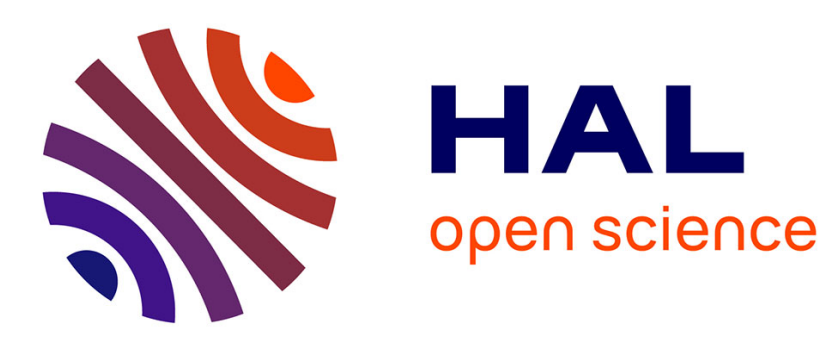

\title{
Cover-Free Codes and Separating System Codes
}

\author{
A.G. d'Yachkov, I.V. Vorobyev, N.A. Polyanskii, V.Yu. Shchukin
}

\section{To cite this version:}

A.G. d'Yachkov, I.V. Vorobyev, N.A. Polyanskii, V.Yu. Shchukin. Cover-Free Codes and Separating System Codes. The 9th International Workshop on Coding and Cryptography 2015 WCC2015, Anne Canteaut, Gaëtan Leurent, Maria Naya-Plasencia, Apr 2015, Paris, France. hal-01276696

\section{HAL Id: hal-01276696 https://inria.hal.science/hal-01276696}

Submitted on 19 Feb 2016

HAL is a multi-disciplinary open access archive for the deposit and dissemination of scientific research documents, whether they are published or not. The documents may come from teaching and research institutions in France or abroad, or from public or private research centers.
L'archive ouverte pluridisciplinaire HAL, est destinée au dépôt et à la diffusion de documents scientifiques de niveau recherche, publiés ou non, émanant des établissements d'enseignement et de recherche français ou étrangers, des laboratoires publics ou privés. 


\title{
Cover-Free Codes and Separating System Codes
}

\author{
A. G. D'yachkov, I.V. Vorobyev, N.A. Polyanskii and V.Yu. Shchukin \\ Lomonosov Moscow State University, Moscow, Russia \\ agd-msu@yandex.ru, vorobyev.i.v@yandex.ru, nikitapolyansky@gmail.com, \\ vpike@mail.ru
}

\begin{abstract}
We discover some important properties of cover-free (CF) codes, separating system (SS) codes and completely separating system (CSS) codes connected with the concept of constant weight CF codes. New upper and lower bounds on the rate of CF and SS codes based on the known results for CF and CSS codes are obtained. Tables of numerical values for the improved upper and lower bounds are presented.
\end{abstract}

Keywords: Cover-free (CF) codes, separating system codes, completely separating system codes, fixed relative weight $\mathrm{CF}$ codes, bounds on the rate

\section{Notations, Definitions and Results}

Let $N, t, s$ and $L$ be integers, $1 \leqslant s<t, 1 \leqslant L \leqslant t-s$, the symbol $\triangleq$ denotes equality by definition, $|A|$ - cardinality of the set $A$, and $[N] \triangleq\{1,2, \ldots, N\}$ - the set of integers from 1 to $N$. The standard symbol $\lfloor a\rfloor(\lceil a\rceil)$ will be used to denote the largest (least) integer $\leqslant a(\geqslant a)$. Introduce a binary matrix $X \triangleq\left\|x_{i}(j)\right\|$, $x_{i}(j)=0,1$ with $t$ columns (codewords) $\boldsymbol{x}(j) \triangleq\left(x_{1}(j), \ldots, x_{N}(j)\right), j \in[t]$, and $N$ rows $\boldsymbol{x}_{i} \triangleq\left(x_{i}(1), \ldots, x_{i}(t)\right), i \in[N]$. Any such matrix is called a binary code $X$ of length $N$ and size $t$. The number of ones in column $\boldsymbol{x}(j)$, i.e., $|\boldsymbol{x}(j)| \triangleq \sum_{i=1}^{N} x_{i}(j)$, is called the weight of $\boldsymbol{x}(j), j \in[t]$. Let $Q, 0<Q<1$, be a fixed parameter. A code $X$ of length $N$ and size $t$ is said to be the constant weight code of the relative weight $Q$ if the weight $|\boldsymbol{x}(j)| \triangleq\lceil Q N\rceil$ for any $j \in[t]$.

\subsection{Cover-Free and Separating Codes}

Let $s \geqslant 1$ and $\ell \geqslant 1$ be positive integers such that $s+\ell \leqslant t$.

Definition 1. [1],[7]. A code $X$ is called a cover-free $(\mathrm{CF})(s, \ell)$-code, if for any two disjoint sets $\mathcal{S}, \mathcal{L} \subset[t],|\mathcal{S}|=s,|\mathcal{L}|=\ell, \mathcal{S} \cap \mathcal{L}=\varnothing$, there exists a row $\boldsymbol{x}_{i}, i \in[N]$, such that

$$
x_{i}(j)=0 \quad \text { for any } j \in \mathcal{S}, \quad \text { and } \quad x_{i}(k)=1 \quad \text { for any } k \in \mathcal{L} .
$$

Taking into account the obvious symmetry over the parameters $s$ and $\ell$, we denote by $t_{c f}(N, s, \ell)=t_{c f}(N, \ell, s)$ the maximal size of $\mathrm{CF}(s, \ell)$-codes of length 
$N$, and by $N_{c f}(t, s, \ell)=N_{c f}(t, \ell, s)$, the minimal length of CF $(s, \ell)$-codes of size $t$. Introduce the rate of $\mathrm{CF}(s, \ell)$-codes:

$$
R_{c f}(s, \ell)=R_{c f}(\ell, s) \triangleq \varlimsup_{N \rightarrow \infty} \frac{\log _{2} t_{c f}(N, s, \ell)}{N}=\varlimsup_{t \rightarrow \infty} \frac{\log _{2} t}{N_{c f}(t, s, \ell)}
$$

The best presently known upper and lower bounds on the rate $R_{c f}(s, \ell)$ of $\mathrm{CF}(s, \ell)$-codes were established in $[11,12]$. If $\ell \geqslant 1$ is fixed and $s \rightarrow \infty$, then these bounds have the following asymptotic form:

$$
\frac{(\ell+1)^{\ell+1}}{e^{\ell+1}} \frac{\log _{2} s}{s^{\ell+1}}(1+o(1)) \leqslant R_{c f}(s, \ell) \leqslant \frac{(\ell+1)^{\ell+1}}{2 e^{\ell-1}} \frac{\log _{2} s}{s^{\ell+1}}(1+o(1)),
$$

where $e$ is the base of the natural logarithm.

Definition 2. [2]. A code $X$ is called a separating system $(s, \ell)$-code or, briefly, SS $(s, \ell)$-code, if for any two disjoint sets $\mathcal{S}, \mathcal{L} \subset[t],|\mathcal{S}|=s,|\mathcal{L}|=\ell$, $\mathcal{S} \cap \mathcal{L}=\varnothing$, there exists a row $\boldsymbol{x}_{i}, i \in[N]$, such that

$$
x_{i}(j)=0 \quad \text { for any } j \in \mathcal{S}, \quad \text { and } \quad x_{i}(k)=1 \quad \text { for any } \quad k \in \mathcal{L},
$$

or

$$
x_{i}(j)=1 \quad \text { for any } j \in \mathcal{S}, \quad \text { and } \quad x_{i}(k)=0 \quad \text { for any } \quad k \in \mathcal{L} .
$$

Taking into account the evident symmetry over the parameters $s$ and $\ell$, denote by $t_{s s}(N, s, \ell)=t_{s s}(N, \ell, s)$ the maximum possible size of SS $(s, \ell)$-codes of length $N$, and denote by $N_{s s}(t, s, \ell)=N_{s s}(t, \ell, s)$ the minimum possible length of SS $(s, \ell)$-code of size $t$. Introduce the rate of SS $(s, \ell)$-codes:

$$
R_{s s}(s, \ell)=R_{s s}(\ell, s) \triangleq \varlimsup_{N \rightarrow \infty} \frac{\log _{2} t_{s s}(N, s, \ell)}{N}=\varlimsup_{t \rightarrow \infty} \frac{\log _{2} t}{N_{s s}(t, s, \ell)}
$$

Definition 3. [3]. Code $X$ is called a completely separating system $(s, \ell)$ code or, briefly, $\operatorname{CSS}(s, \ell)$-code, if for any two disjoint sets $\mathcal{S}, \mathcal{L} \subset[t],|\mathcal{S}|=s$, $|\mathcal{L}|=\ell, \mathcal{S} \cap \mathcal{L}=\varnothing$, there exist two rows $\boldsymbol{x}_{i}, \boldsymbol{x}_{j}, i, j \in[N]$, such that

$$
x_{i}(m)=0 \quad \text { for any } \quad m \in \mathcal{S}, \quad \text { and } \quad x_{i}(k)=1 \quad \text { for any } \quad k \in \mathcal{L},
$$

and

$$
x_{j}(m)=1 \quad \text { for any } m \in \mathcal{S}, \quad \text { and } \quad x_{j}(k)=0 \text { for any } k \in \mathcal{L} \text {. }
$$

Given the symmetry over $s$ and $\ell$, denote by $t_{c s s}(N, s, \ell)=t_{c s s}(N, \ell, s)$ the maximum size of CSS $(s, \ell)$-codes of length $N$, and by $N_{c s s}(t, s, \ell)=N_{c s s}(t, \ell, s)$, the minimum length of CSS $(s, \ell)$ codes of size $t$. Introduce the rate of CSS $(s, \ell)$ codes:

$$
R_{c s s}(s, \ell)=R_{c s s}(\ell, s) \triangleq \varlimsup_{N \rightarrow \infty} \frac{\log _{2} t_{c s s}(N, s, \ell)}{N}=\varlimsup_{t \rightarrow \infty} \frac{\log _{2} t}{N_{c s s}(t, s, \ell)} .
$$


Bounds on the rates (2)-(3) along with constructions of SS $(s, \ell)$-codes and CSS $(s, \ell)$-codes have been investigated in many papers. See, overviews $[4,5]$. Note the evident

Proposition 1. [4,5]. (Monotonicity properties). For any $s \geqslant 1$ and $\ell \geqslant 1$, the rate $R_{c f}(s, s)=R_{c s s}(s, s)$ and the inequalities

$$
R_{s s}(s, l) / 2 \leqslant R_{c s s}(s, l) \leqslant R_{c f}(s, l) \leqslant R_{s s}(s, l)
$$

hold.

In Definitions 1-3, we follow the notations used in the survey [5]. The aim of our paper is presented in the Abstract.

\subsection{Applications of Separating Codes}

A separating code is very natural combinatorial object. It has applications in such fields as automata synthesis, technical diagnosis and the construction of hash functions.

We briefly describe the application of separating codes in digital fingerprinting (see [15],[16]). Vendor marks each copy of digital object with an unique key. Coalition of dishonest users can compare their copies and find bits, where the copies differs. These bits must be a part of fingerprint. By changing these bits they are able to create a pirate copy. Using separating $(s, 1)$-codes, which are also known as frameproof codes, as a keys, we can guarantee that a coalition of at most $s$ users cannot produce a valid key.

\subsection{Constant Weight CF $(s, \ell)-\operatorname{codes}$}

Denote by $t_{c f}^{Q}(N, s, \ell)=t_{c f}^{1-Q}(N, \ell, s)$ the maximum possible size of constant weight $\mathrm{CF}(s, \ell)$-codes of length $N$ and the relative weight $Q$. Denote by $N_{c f}^{Q}(t, s, \ell)=N_{c f}^{1-Q}(t, \ell, s)$ the minimum possible length of constant weight $\mathrm{CF}(s, \ell)$-codes of size $t$ and the relative weight $Q$. Introduce the concept of $Q$-rate of $\mathrm{CF}(s, \ell)$-codes:

$$
R_{c f}^{Q}(s, \ell)=R_{c f}^{Q}(\ell, s) \triangleq \varlimsup_{N \rightarrow \infty} \frac{\log _{2} t_{c f}^{Q}(N, s, \ell)}{N}=\varlimsup_{t \rightarrow \infty} \frac{\log _{2} t}{N_{c f}^{Q}(t, s, \ell)} .
$$

Proposition 2. The $Q$-rate of $C F(s, \ell)$-codes $R_{c f}^{Q}(s, \ell)$ and the rate $R_{c f}(s, \ell)$ of $C F(s, \ell)$-codes satisfy the inequalities:

$$
R_{c f}^{Q}(s+1, \ell) \leq(1-Q) \cdot R_{c f}(s, \ell), \quad R_{c f}^{Q}(s, \ell+1) \leq Q \cdot R_{c f}(s, \ell) .
$$

Proof. Consider an arbitrary constant weight CF $(s+1, \ell)$-code $X$ of length $N$, size $t$ and the relative weight $Q$. Fix an arbitrary column $\boldsymbol{x}(j)$. Delete the column $\boldsymbol{x}(j)$ and all $\lceil Q N\rceil$ rows having ones in $\boldsymbol{x}(j)$. It's easy to see that the obtained code $X^{\prime}$ is a CF $(s, \ell)$-code of size $t-1$ and length $\leqslant(1-Q) N$. This yields

$$
N_{c f}^{Q}(t, s+1, \ell) \cdot(1-Q) \geqslant N_{c f}(t-1, s, \ell) .
$$


Therefore, the rate definitions (2) and (5) lead to the first inequality in (6). The second inequality in (6) is established in the similar way.

Proposition 3. The rate of $S S(s, \ell)$-codes $R_{s s}(s, \ell)$ and the $1 / 2$-rate of $C F(s, \ell)$-codes $R_{c f}^{1 / 2}(s, \ell)$ satisfy the inequality

$$
R_{s s}(s, \ell) \leqslant 2 \cdot R_{c f}^{1 / 2}(s, \ell) .
$$

Proof. Consider an arbitrary SS $(s, \ell)$-code $X$ of size $t$ and length $N$. Construct the code $X^{\prime}=\left(\boldsymbol{x}^{\prime}(1), \boldsymbol{x}^{\prime}(2), \ldots, \boldsymbol{x}^{\prime}(t)\right)$ of size $t$ and length $2 N$ as follows: $\boldsymbol{x}^{\prime}(i)=\boldsymbol{x}(i) \& \overline{\boldsymbol{x}(i)}, i \in[t]$, where the symbol \& denotes the concatenation of two vectors, and $\overline{\boldsymbol{x}(i)} \triangleq\left(\overline{x_{1}(i)}, \overline{x_{2}(i)}, \ldots, \overline{x_{N}(i)}\right)$ denotes the opposite vector to $\boldsymbol{x}(i)$. One can easily see that the code $X^{\prime}$ is a constant weight CF $(s, \ell)$-code of the relative weight $1 / 2$. Hence, the rate definitions (2) and (5) lead to (7).

Our new upper bounds on the rate of $S S(s, \ell)$-codes are obtained with the help of the known upper bounds on the rate $R_{c f}(s, \ell)$ of $\mathrm{CF}(s, \ell)$-codes and the following

Theorem 1. The rate $R_{s s}(s, \ell)$ of $S S(s, \ell)$-codes and the rate $R_{c f}(s, \ell)$ of $C F(s, \ell)$-codes satisfy inequalities

$$
\begin{aligned}
& R_{c f}(s, \ell) \leqslant R_{s s}(s, \ell) \leqslant R_{c f}(s-1, \ell), \quad \ell \geqslant 1, s \geqslant 2, \\
& R_{c f}(s, \ell) \leqslant R_{s s}(s, \ell) \leqslant R_{c f}(s, \ell-1), \quad \ell \geqslant 2, s \geqslant 1 .
\end{aligned}
$$

Proof. The left-hand side inequalities in (8) follow immediately from (4). To prove the right-hand side inequalities in (8), we consequently apply (7) and (6) for $Q=1 / 2$.

In particular, Theorem 1 implies that the rate $R_{s s}(s, \ell)$ of $S S(s, \ell)$-codes and the rate $R_{c f}(s, \ell)$ of $\mathrm{CF}(s, \ell)$-codes satisfy the same asymptotic inequalities (1).

\subsection{Recurrent Inequalities}

The best known upper bounds [7]-[9] on the rate $R_{c f}(s, \ell)$ of $\mathrm{CF}(s, \ell)$-codes are based on the recurrent inequality $[6]$ :

$$
R_{c f}(s, \ell) \leqslant R_{c f}(s-u, \ell-v) \cdot \frac{u^{u} v^{v}}{(u+v)^{u+v}}, \quad 1 \leqslant u \leqslant s-1, \quad 1 \leqslant v \leqslant \ell-1
$$

and its improvement [10]:

$$
R_{c f}(s, \ell) \leqslant \frac{R_{c f}(s-u, \ell-v)}{R_{c f}(s-u, \ell-v)+\frac{(u+v)^{u+v}}{u^{u} v^{v}}}, \quad 1 \leqslant u \leqslant s-1, \quad 1 \leqslant v \leqslant \ell-1 .
$$

The similar joint recurrent inequalities for the rates $R_{c f}(s, \ell), R_{s s}(s, \ell)$ and $R_{c s s}(s, \ell)$ are formulated below in the form of Theorem 2 which will be established in Sect. 2.

Theorem 2. 1) For any $u \in[s-1], v \in[\ell-1], u \neq v$,

$$
R_{s s}(s, \ell) \leqslant R_{s s}(s-u, \ell-v) \cdot \max _{0 \leqslant z \leqslant 1}\left\{z^{u}(1-z)^{v}+(1-z)^{u} z^{v}\right\} .
$$


2) For any $v \in[\ell-1]$,

$$
R_{s s}(s, \ell) \leqslant R_{c s s}(s-v, \ell-v) \frac{1}{2^{2 v-1}} .
$$

3) For any $v \in[\ell-1]$ and $u=v+s-\ell$,

$$
R_{s s}(s, \ell) \leqslant R_{c s s}(s-u, \ell-v) \cdot \max _{0 \leqslant z \leqslant 1}\left\{z^{u}(1-z)^{v}+(1-z)^{u} z^{v}\right\} .
$$

4) For any $i \in[s-1]$,

$$
R_{s s}(s, s) \leqslant \frac{R_{c f}(i, i)}{2^{2 s-2 i-1}}
$$

5) For any $v \in[\ell-1]$,

$$
R_{c f}(s, \ell) \leqslant R_{c s s}(s-v, \ell-v) \frac{1}{2^{2 v}} .
$$

6) For any $v \in[\ell-1]$ and $u=v+s-\ell$,

$$
R_{c f}(s, \ell) \leqslant R_{c s s}(s-u, \ell-v) \cdot \frac{u^{u} v^{v}}{(u+v)^{u+v}}
$$

Note that the monotonicity inequality (4) and (16) imply a possibility to improve the recurrent inequalities (9)-(10). In Sect. 1.5, we present detailed Tables of new upper bounds on the rates $R_{c f}(s, \ell), R_{s s}(s, \ell)$ and $R_{c s s}(s, \ell)$ which follow from Theorems 1-2.

\subsection{Tables of Upper Bounds}

In Table 1, we present the best known upper bounds [5] on the rate of CSS $(s, \ell)$-codes. We use these values to improve upper bounds on the rates of $\mathrm{CF}$ $(s, \ell)$-codes and SS $(s, \ell)$-codes with the help of Theorem 2.

Table 1. Upper Bounds for Completely Separating System $(s, \ell)$-Codes

\begin{tabular}{|c|c|c|c|c|c|c|}
\hline$s \mid \ell$ & 1 & 2 & 3 & 4 & 5 & 6 \\
\hline 1 & 1 & 0.322 & 0.199 & 0.14 & 0.106 & 0.083 \\
2 & 0.322 & 0.161 & 0.0662784 & 0.0429588 & 0.0286 & 0.0203 \\
3 & 0.199 & 0.0662784 & 0.0353515 & 0.0153287 & 0.0101062 & 0.00669 \\
4 & 0.14 & 0.0429588 & 0.0153287 & 0.00836963 & 0.00370404 & 0.00245936 \\
5 & 0.106 & 0.0286 & 0.0101062 & 0.00370404 & 0.00204224 & 0.000911804 \\
6 & 0.083 & 0.0203 & 0.00669 & 0.00245936 & 0.000911804 & 0.000504899 \\
\hline
\end{tabular}

In Table 2, upper bounds on the rate of $\mathrm{CF}(s, \ell)$-codes are given.

In Table 3 , we provide new upper bounds for SS $(s, \ell)$-code. 
Table 2. Upper Bounds for Cover-Free $(s, \ell)$-Codes

\begin{tabular}{|c|c|c|c|c|c|c|}
\hline$s \mid \ell$ & 1 & 2 & 3 & 4 & 5 & 6 \\
\hline 1 & 1 & $0.322^{1}$ & $0.199^{1}$ & $0.14^{1}$ & $0.106^{1}$ & $0.083^{1}$ \\
2 & $0.322^{1}$ & $0.161^{1}$ & $0.0744^{2}$ & $0.0455^{2}$ & $0.0286^{2}$ & $0.0203^{2}$ \\
3 & $0.199^{1}$ & $0.0744^{2}$ & $0.035352^{3}$ & $0.016570^{4}$ & $0.010740^{4}$ & $0.006690^{2}$ \\
4 & $0.14^{1}$ & $0.0455^{2}$ & $0.016570^{4}$ & $0.008370^{3}$ & $0.003832^{4}$ & $0.002527^{4}$ \\
5 & $0.106^{1}$ & $0.0286^{2}$ & $0.010740^{4}$ & $0.003832^{4}$ & $0.002042^{3}$ & $0.000926^{4}$ \\
6 & $0.083^{1}$ & $0.0203^{2}$ & $0.00669^{2}$ & $0.002527^{4}$ & $0.000926^{4}$ & $0.000505^{3}$ \\
\hline
\end{tabular}

1 See [7]. ${ }^{2}$ See [10]. ${ }^{3}$ See [5]. ${ }^{4}$ Statement 5 of Theorem 2.

Table 3. Upper Bounds for Separating Systems $(s, \ell)$-Codes

\begin{tabular}{|c|c|c|c|c|c|c|}
\hline$s \mid \ell$ & 1 & 2 & 3 & 4 & 5 & 6 \\
\hline 1 & 1 & $0.5^{3}$ & $0.322^{1}$ & $0.199^{1}$ & $0.14^{1}$ & $0.106^{1}$ \\
2 & $0.5^{3}$ & $0.283477^{3}$ & $0.120209^{3}$ & $0.0744^{1}$ & $0.0455^{1}$ & $0.0286^{1}$ \\
3 & $0.322^{1}$ & $0.120209^{3}$ & $0.0662784^{3}$ & $0.029511^{3}$ & $0.0183^{1}$ & $0.0109^{1}$ \\
4 & $0.199^{1}$ & $0.0744^{1}$ & $0.029511^{3}$ & $0.0163042^{3}$ & $0.00728895^{3}$ & $0.00441894^{2}$ \\
5 & $0.14^{1}$ & $0.0455^{1}$ & $0.0183^{1}$ & $0.00728895^{3}$ & $0.00403793^{3}$ & $0.00181049^{3}$ \\
6 & $0.106^{1}$ & $0.0286^{1}$ & $0.0109^{1}$ & $0.00441894^{2}$ & $0.00181049^{3}$ & $0.00100459^{3}$ \\
\hline
\end{tabular}

Let us demonstrate how these values have been obtained. Consider, for instance, upper bound for SS $(4,6)$-code. Applying Statement 3 of Theorem 2 with $v=3$ and $u=1$, we obtain the following inequality

$$
R_{s s}(4,6) \leqslant R_{c s s}(3,3) \max _{0 \leqslant z \leqslant 1}\left\{z(1-z)^{3}+(1-z) z^{3}\right\} .
$$

The maximum value $\frac{1}{8}$ of $z(1-z)^{3}+(1-z) z^{3}$ is attained at $z=\frac{1}{2}$. Hence, the rate

$$
R_{s s}(4,6) \leqslant \frac{R_{c s s}(3,3)}{8} \leqslant \frac{0.0353515}{8} \approx 0.00441894 .
$$

It is clear that this bound is better than the previous one 0.00485634 , computed by Theorem 5 in [5].

\subsection{Tables of Lower Bounds}

In Table 4, we remind the best known lower bounds on the rate of $\mathrm{CF}(s, \ell)$ codes $[11,12]$.

With the help of these values and the inequality (4) we improve lower bounds for SS $(s, \ell)$-codes, which are presented in Table 5 . 
Table 4. Lower Bounds for Cover-Free $(s, \ell)$-codes

\begin{tabular}{|c|c|c|c|c|c|c|}
\hline$s \mid \ell$ & 1 & 2 & 3 & 4 & 5 & 6 \\
\hline 1 & 1 & 0.182 & 0.082 & 0.0566 & 0.042 & 0.0325 \\
2 & 0.182 & 0.0584 & 0.031 & 0.0185 & 0.012 & 0.00825 \\
3 & 0.082 & 0.031 & 0.00978 & 0.00553 & 0.00336 & 0.00215 \\
4 & 0.0566 & 0.0185 & 0.00553 & 0.00192 & 0.0011 & 0.000671 \\
5 & 0.042 & 0.012 & 0.00336 & 0.0011 & 0.000404 & 0.000234 \\
6 & 0.0325 & 0.00825 & 0.00215 & 0.000671 & 0.000234 & 0.000088 \\
\hline
\end{tabular}

Table 5. Lower Bounds for Separating Systems $(s, \ell)$-codes

\begin{tabular}{|c|c|c|c|c|c|c|}
\hline$s \mid \ell$ & 1 & 2 & 3 & 4 & 5 & 6 \\
\hline 1 & 1 & $0.2075^{3}$ & $0.082^{1}$ & $0.0566^{1}$ & $0.042^{1}$ & $0.0325^{1}$ \\
2 & $0.2075^{3}$ & $0.0642^{2}$ & $0.031^{1}$ & $0.0185^{1}$ & $0.012^{1}$ & $0.00825^{1}$ \\
3 & $0.082^{1}$ & $0.031^{1}$ & $0.00978^{1}$ & $0.00553^{1}$ & $0.00336^{1}$ & $0.00215^{1}$ \\
4 & $0.0566^{1}$ & $0.0185^{1}$ & $0.00553^{1}$ & $0.00192^{1}$ & $0.0011^{1}$ & $0.000671^{1}$ \\
5 & $0.042^{1}$ & $0.012^{1}$ & $0.00336^{1}$ & $0.0011^{1}$ & $0.000404^{1}$ & $0.000234^{1}$ \\
6 & $0.0325^{1}$ & $0.00825^{1}$ & $0.00215^{1}$ & $0.000671^{1}$ & $0.000234^{1}$ & $0.000088^{1}$ \\
\hline
\end{tabular}

$1 \quad$ See Theorem 1 and $[12,11] . \quad 2 \quad$ See $[13] . \quad{ }^{3} \quad$ See $[4,14]$.

\section{Proof of Theorem 2}

Denote by $\mathcal{P}_{u}(t)$ all $u$-subsets $t$-set, i.e. $\mathcal{P}_{u}(t) \triangleq\{P \subset[t]: \quad|P|=u\}$. Without loss of generality we suppose that $s \geqslant \ell$.

Proof of Statement 1. Let $\mathcal{U} \subset[t],|\mathcal{U}|=u$, and $\mathcal{V} \subset[t],|\mathcal{V}|=v$, $\mathcal{U} \cap \mathcal{V}=\varnothing$ be two disjoint subsets of $t$-set with cardinalities $u$ and $v$ respectively. Denote by $X$ an arbitrary binary code of size $t$ and length $N$. Define the set of rows $D_{u, v}(\mathcal{U}, \mathcal{V}, X) \subset[N], 0 \leqslant\left|D_{u, v}(\mathcal{U}, \mathcal{V}, X)\right| \leqslant N$, as the set of rows $\boldsymbol{x}_{i}$ of the code $X$ such that one of the conditions

$$
\begin{array}{lll}
x_{i}(j)=0 \text { for any } j \in \mathcal{U} \text { and } & x_{i}(k)=1 \text { for any } k \in \mathcal{V}, \\
x_{i}(j)=1 \text { for any } j \in \mathcal{U} & \text { and } & x_{i}(k)=0 \text { for any } k \in \mathcal{V}
\end{array}
$$

holds. Define the average number and the maximum

$$
\bar{D}_{u, v}(X) \triangleq \sum_{\substack{\mathcal{U} \in \mathcal{P}_{u}(t), \mathcal{V} \in \mathcal{P}_{v}(t), \mathcal{U} \cap \mathcal{V}=\varnothing}} \frac{\left|D_{u, v}(\mathcal{U}, \mathcal{V}, X)\right|}{\left(\begin{array}{c}
t \\
u+v
\end{array}\right) \cdot\left(\begin{array}{c}
u+v \\
u
\end{array}\right)}, \quad \bar{D}_{u, v}(t, N)=\max _{X} \bar{D}_{u, v}(X),
$$

where the maximum is taken over all codes $X$ of length $N$ and size $t$.

Lemma 1. The number $\bar{D}_{u, v}(t, N)$ satisfies the asymptotic inequality

$$
\varlimsup_{t \rightarrow \infty} \frac{\bar{D}_{u, v}(t, N)}{N} \leqslant \max _{0 \leqslant z \leqslant 1}\left\{z^{u}(1-z)^{v}+(1-z)^{u} z^{v}\right\} .
$$


Proof of Lemma 1. Let $\mathcal{K} \subset[t],|\mathcal{K}|=u+v$ and $i \in N$. Denote by $\boldsymbol{x}_{i}(K)$ the $1 \times(u+v)$ submatrix of $X$ composed of elements of the $i$-th row and columns from the set $\mathcal{K}$. Define

$$
I(X, \mathcal{K}, i) \triangleq \begin{cases}1 & \text { if } x_{i}(\mathcal{K}) \text { contains either } u \text { zeroes or } v \text { zeroes, } \\ 0 & \text { otherwise. }\end{cases}
$$

Denote by $M_{u, v}(X)$ the number of all possible $1 \times(u+v)$ submatrices of $X$ with either $u$ zeroes and $v$ ones or $v$ zeroes and $u$ ones, i.e.

$$
M_{u, v}(X) \triangleq \sum_{i \in N, \mathcal{K} \in \mathcal{P}_{u+v}(t)} I(X, \mathcal{K}, i) .
$$

Let $a_{i}\left(t-a_{i}\right)$ be equal to the number of zeroes (ones) in the $i$-th row of the code $X$. Then

$$
M_{u, v}(X)=\sum_{i=1}^{N}\left(\begin{array}{c}
a_{i} \\
u
\end{array}\right) \cdot\left(\begin{array}{c}
t-a_{i} \\
v
\end{array}\right)+\sum_{i=1}^{N}\left(\begin{array}{c}
a_{i} \\
v
\end{array}\right) \cdot\left(\begin{array}{c}
t-a_{i} \\
u
\end{array}\right) .
$$

On the other hand

$$
M_{u, v}(X)=\bar{D}_{u, v}(X) \cdot\left(\begin{array}{c}
t \\
u+v
\end{array}\right)\left(\begin{array}{c}
u+v \\
u
\end{array}\right) .
$$

These two equations lead to

$$
\left(\begin{array}{c}
t \\
u+v
\end{array}\right)\left(\begin{array}{c}
u+v \\
u
\end{array}\right) \cdot \bar{D}_{u, v}(X) \leqslant N \cdot \max _{a \in[t]}\left\{\left(\begin{array}{l}
a \\
u
\end{array}\right) \cdot\left(\begin{array}{c}
t-a \\
v
\end{array}\right)+\left(\begin{array}{l}
a \\
v
\end{array}\right) \cdot\left(\begin{array}{c}
t-a \\
u
\end{array}\right)\right\} .
$$

If $t \rightarrow \infty$, then the passage to the limit yields (17). Lemma 1 is proved.

To complete the proof of Statement 1 we need

Lemma 2. For any $u \in[s-1]$ and $v \in[\ell-1]$, the minimum length of $S S$ $(s-u, \ell-v)$-code of size $t$ satisfies the inequality

$$
N_{s s}(t-(u+v), s-u, \ell-v) \leqslant \bar{D}_{u, v}(t, N) .
$$

Proof of Lemma 2. Let $X$ be an arbitrary SS $(s, \ell)$-code of size $t$ and length $N$. Consider two disjoint sets $\mathcal{U} \subset[t],|\mathcal{U}|=u, \mathcal{V} \subset[t],|\mathcal{V}|=v$, $\mathcal{U} \cap \mathcal{V}=\varnothing$, such that $\left|D_{u, v}(\mathcal{U}, \mathcal{V}, X)\right| \geqslant \bar{D}_{u, v}(X)$. Obviously, we can find such sets, since the number $\bar{D}_{u, v}(X)$ is equal to the average value of $\left|D_{u, v}(\mathcal{U}, \mathcal{V}, X)\right|$ over all $\mathcal{U}$ and $\mathcal{V}$ by definition. Define the code $X^{\prime}$ of length $\left|D_{u, v}(\mathcal{U}, \mathcal{V}, X)\right|$ and size $t-(u+v)$ as the subcode of $X$ composed of rows $D_{u, v}(\mathcal{U}, \mathcal{V}, X)$ and columns $[t] \backslash\{\mathcal{U} \cup \mathcal{V}\}$. Let us show that $X^{\prime}$ is an SS $(s-u, \ell-v)$-code. Indeed, fix any two sets $\mathcal{U}^{\prime} \subset[t-(u+v)],\left|\mathcal{U}^{\prime}\right|=s-u$, and $\mathcal{V}^{\prime} \subset[t-(u+v)],\left|\mathcal{V}^{\prime}\right|=\ell-v$, $\mathcal{U}^{\prime} \cap \mathcal{V}^{\prime}=\varnothing$. Then find the columns in $X$ corresponding to $\mathcal{U}^{\prime}$ and $\mathcal{V}^{\prime}$. Denote these columns by $\hat{\mathcal{U}}^{\prime}$ and $\hat{\mathcal{V}}^{\prime}$ respectively. Note that these sets don't intersect $\mathcal{U}$ and $\mathcal{V}$ by construction of the code $X^{\prime}$. Hence, for the SS $(s, \ell)$-code $X$ and sets 
$\hat{\mathcal{U}}^{\prime} \cup \mathcal{U},\left|\hat{\mathcal{U}}^{\prime} \cup \mathcal{U}\right|=s$, and $\mathcal{V} \cup \hat{\mathcal{V}}^{\prime},\left|\mathcal{V} \cup \hat{\mathcal{V}}^{\prime}\right|=\ell$, there exists a row $\boldsymbol{x}_{i}$ in $X$, such that one of the conditions

$$
\begin{aligned}
& x_{i}(j)=0 \quad \text { for any } j \in \hat{\mathcal{U}}^{\prime} \cup \mathcal{U}, \quad \text { and } \quad x_{i}(k)=1 \quad \text { for any } \quad k \in \mathcal{V} \cup \hat{\mathcal{V}}^{\prime} \text {, } \\
& x_{i}(j)=1 \quad \text { for any } j \in \hat{\mathcal{U}}^{\prime} \cup \mathcal{U}, \quad \text { and } \quad x_{i}(k)=0 \text { for any } k \in \mathcal{V} \cup \hat{\mathcal{V}}^{\prime}
\end{aligned}
$$

holds. Note that this row belongs to the set $D_{u, v}(\mathcal{U}, \mathcal{V}, X)$. Therefore, the code $X^{\prime}$ is an SS $(s-u, \ell-v)$-code. Lemma 2 is proved.

For $N \triangleq N_{s s}(t, s, \ell)$, the inequality (18) of Lemma 2 can be written as:

$$
\frac{N_{s s}(t-(u+v), s-u, \ell-v)}{N_{s s}(t, s, \ell)} \leqslant \frac{\bar{D}_{u, v}(t, N)}{N}, \quad N=N_{s s}(t, s, \ell) .
$$

If $t \rightarrow \infty$, then in virtue of (17), the passage to the limit in (19) yields

$$
\begin{aligned}
\frac{R_{s s}(s, \ell)}{R_{s s}(s-u, \ell-v)} \leqslant & \varlimsup_{t \rightarrow \infty} \frac{N_{s s}(t-(u+v), s-u, \ell-v)}{N_{s s}(t, s, \ell)} \leqslant \varlimsup_{t \rightarrow \infty} \frac{\bar{D}_{u, v}(t, N)}{N} \leqslant \\
& \leqslant \max _{0 \leqslant z \leqslant 1}\left\{z^{u}(1-z)^{v}+(1-z)^{u} z^{v}\right\} .
\end{aligned}
$$

Statement 1 is proved completely.

Proof of Statement 2. The proof of Statement 2 is similar to the proof of Statement 1, but instead of Lemma 2 we need

Lemma 3. For any $v \in[\ell-1]$ length of $C S S(s-v, \ell-v)$-code satisfies the inequality $N_{c s s}(t-2 v, s-v, \ell-v) \leqslant \bar{D}_{v, v}(t, N)$.

Proof of Lemma 3. Consider two sets $\mathcal{U} \subset[t],|\mathcal{U}|=v$, and $\mathcal{V} \subset[t]$, $|\mathcal{V}|=v, \mathcal{U} \cap \mathcal{V}=\varnothing$, such that the inequality $\left|D_{v, v}(\mathcal{U}, \mathcal{V}, X)\right| \geqslant \bar{D}_{v, v}(X)$ holds. Consider the subcode of $X$ composed of columns corresponding to sets $\mathcal{U}$ and $\mathcal{V}$. Without loss of generality, we assume that each row from $D_{v, v}(\mathcal{U}, \mathcal{V}, X)$ has the following form

$$
00 \ldots 011 \ldots 1 \text {. }
$$

Define the code $X^{\prime}$ of length $\left|D_{v, v}(\mathcal{U}, \mathcal{V}, X)\right|$ and size $t-2 v$ as the subcode of $X$ composed of rows $D_{v, v}(\mathcal{U}, \mathcal{V}, X)$ and columns $[t] \backslash\{\mathcal{U} \cup \mathcal{V}\}$. Let us prove that $X^{\prime}$ is an CSS $(s-v, \ell-v)$-code. Indeed, fix any two sets $\mathcal{U}^{\prime} \subset[t-2 v],\left|\mathcal{U}^{\prime}\right|=s-v$, and $\mathcal{V}^{\prime} \subset[t],\left|\mathcal{V}^{\prime}\right|=\ell-v, \mathcal{U}^{\prime} \cap \mathcal{V}^{\prime}=\varnothing$. Then find the columns in $X$ corresponding to $\mathcal{U}^{\prime}$ and $\mathcal{V}^{\prime}$. Denote them by $\hat{\mathcal{U}}^{\prime}$ and $\hat{\mathcal{V}}^{\prime}$ respectively. These two sets don't intersect $\mathcal{U}$ and $\mathcal{V}$ by construction of $X^{\prime}$. Hence for the SS $(s, \ell)$-code $X$ and sets $\hat{\mathcal{U}}^{\prime} \cup \mathcal{U}$, $\left|\hat{\mathcal{U}}^{\prime} \cup \mathcal{U}\right|=s$, and $\mathcal{V} \cup \hat{\mathcal{V}}^{\prime},\left|\mathcal{V} \cup \hat{\mathcal{V}}^{\prime}\right|=\ell$, there exists a row $\boldsymbol{x}_{i}$ in $X$, such that

$$
x_{i}(j)=0 \quad \text { for any } j \in \hat{\mathcal{U}}^{\prime} \cup \mathcal{U}, \quad \text { and } \quad x_{i}(k)=1 \quad \text { for any } \quad k \in \mathcal{V} \cup \hat{\mathcal{V}}^{\prime} .
$$

For sets $\mathcal{U} \cup \hat{\mathcal{V}}^{\prime}$ and $\mathcal{V} \cup \hat{\mathcal{U}}^{\prime}$ we also can find such row. Note that these rows belong to $D_{v, v}(\mathcal{U}, \mathcal{V}, X)$. Therefore, the code $X^{\prime}$ is an CSS $(s-v, \ell-v)$-code.

Lemma 3 and Statement 2 are proved.

Proof of Statement 3. Taking into account the equality $s-u=l-v$, the proof of (13) is essentially the same as the proof of (12). 
Proof of Statements 4-6. If we apply the second claim (12) to the particular case $v=s-i$, then the recurrent inequality (14) immediately follows from the evident property: $\max _{0 \leqslant z \leqslant 1}\left\{z^{v}(1-z)^{v}\right\}=(1 / 2)^{2 s-2 i}$.

The recurrent inequalities (15)-(16) can be easily obtained with the help of the same arguments that were used to establish the recurrent inequalities (12)(13).

Theorem 2 is proved completely.

\section{References}

1. Mitchell, C.J. and Piper, F.C., "Key Storage in Secure Networks", Discrete Appl. Math., vol. 21, no. 3, pp. 215-228, 1988.

2. A. D. Friedman, R. L. Graham, and J. D. Ullman, "Universal single transition time asynchronous state assignments", IEEE Trans. Comput., vol. 18, no. 6, pp. 541$547,1969$.

3. Mago G. "Monotone Functions in Sequential Circuits", IEEE Trans. Comput., v. 22, no. 10, pp. 928-933, 1973.

4. Yu. L. Sagalovich, "Separating systems", Problems of Information Transmission, vol. 30, no. 2,pp. 105-123, 1994.

5. G. D. Cohen and H. G. Schaathun, "Asymptotic overview on separating codes", Tech. Report 248, Department of Informatics, University of Bergen, Bergen, Norway, 2003.

6. Engel, K., "Interval Packing and Covering in the Boolean Lattice", Combin. Probab. Comput., vol. 5, no. 4, pp. 373-384, 1996.

7. D'yachkov A.G., Vilenkin P.A., Macula A.J., Torney D.C., "Families of Finite Sets in Which No Intersection of $\ell$ Sets Is Covered by the Union of $s$ Others", Journal of Combinatorial Theory, Series A, vol. 99. pp. 195-218, 2002.

8. D'yachkov A.G., Vilenkin P.A., Macula A.J., Torney D.C., Yekhanin S.M., "New Results in the Theory of Superimposed Codes", Proc. Seventh Int. Workshop on Algebraic and Combinatorial Coding Theory, Bansko, Bulgaria, pp. 126-136, 2000.

9. D'yachkov A.G., Rykov V.V., Deppe C., Lebedev V.S. "Superimposed Codes and Threshold Group Testing", Information Theory, Combinatorics, and Search Theory, Lecture Notes in Computer Science, vol. 7777, pp. 509-533, 2013.

10. Lebedev, V.S., "Asymptotic Upper Bound for the Rate of (w, r) Cover-Free Codes", Problems of Information Transmission, vol. 39, no. 4, pp. 317-323, 2003.

11. D'yachkov A.G., Vorobyev I.V., Polyanskii N.A., Shchukin V.Yu., "Bounds on the Rate of Disjunctive Codes", Problems of Information Transmission, vol. 50, no. 1, pp. 27-56, 2014.

12. D'yachkov A.G., Vorobyev I.V., Polyanskii N.A., Shchukin V.Yu., "Bounds on the Rate of Superimposed Codes", 2014 IEEE International Symposium on Information Theory, pp. 2341-2345, Honolulu, HI USA, Jun.29-Jul.4, 2014.

13. Yu. L. Sagalovich, "Completely Separating Systems", Probl. Peredachi Inf., vol. 18, no. 2, pp. 74-82, 1982.

14. Gerard Cohen and Gilles Zemor, "Intersecting codes and independent families", IEEE Trans. Inform. Theory, 40:1872-1881, 1994.

15. Dan Boneh and James Shaw. "Collusion-secure fingerprinting for digital data", IEEE Trans. Inform. Theory, 44(5):1897?1905, 1998.

16. J. N. Staddon, D. R. Stinson and R. Wei. "Combinatorial properties of frameproof and traceability codes", IEEE Trans. Inform. Theory, 47:1042-1049, 2001. 